\title{
Advanced public transport system scheduling and control
}

\author{
Hong K. Lo • William H.K. Lam • S.C. Wong • \\ Janny M.Y. Leung
}

Published online: 22 February 2011

C) Springer-Verlag 2011

This special issue continues the previous issue of the journal Public Transport. It is based on papers selected from the 11th International Conference on Advanced Systems for Public Transport (CASPT) held in Hong Kong on 20-22 July 2009. CASPT serves as a forum for the international community of researchers and practitioners on all aspects of public transport planning and operations, covering significant contributions to the theory and application of systems and methodologies for advancing public transport planning and operations.

Five papers are collected in this special issue, focusing on crew and vehicle scheduling, and train and runway control. This special issue provides a timely review of recent efforts in extending the consideration of public transport scheduling and control for incident scenarios, centralized versus distributed systems, accommodat-

H.K. Lo (凶)

Department of Civil and Environmental Engineering, The Hong Kong University of Science and Technology, Clear Water Bay, Kowloon, Hong Kong

e-mail: cehklo@ust.hk

W.H.K. Lam

Department of Civil and Structural Engineering, The Hong Kong Polytechnic University, Yuk Choi Road, Hung Hom, Kowloon, Hong Kong

e-mail: cehklam@polyu.edu.hk

S.C. Wong

Department of Civil Engineering, The University of Hong Kong, Pokfulam Road, Hong Kong e-mail: hhecwsc@hkucc.hku.hk

J.M.Y. Leung

Department of Systems Engineering and Engineering Management, The Chinese University of Hong Kong, Shatin, N.T., Hong Kong

e-mail: janny@se.cuhk.edu.hk 
ing driver shift preferences and conflicting management objectives, and joint traffic control and bus scheduling.

The paper by Abbink, Mobach, Fioole, Kroon, van der Heijden, Wijngaards presents a method based on multi-agent techniques to solve the train driver rescheduling problem in case of a large disruption, assuming that the timetable and rolling stock have already been rescheduled based on an incident scenario. The method was tested on several realistic disruption instances of Netherlands Railways, showing promising results.

The paper by Corman, D'Ariano, Pacciarelli, and Pranzo presents a novel distributed optimization system for train control on a Dutch railway network. Extensive computational assessments of the centralized and distributed systems are performed by using simple and advanced train scheduling algorithms, including dispatching rules adopted during operations. The effectiveness of the distributed system is demonstrated in terms of computation time and delay minimization.

The paper by Montalva, Muñoz, and Paredes investigates and compares different shift assignment policies of transit agencies, considering the approximate utility of each shift to drivers. The study shows that flexible shifts offer a win-win opportunity for the transit agency and drivers, with improved productivity and job satisfaction. This result is particularly relevant since transit operational costs are strongly dependent on their labor force.

The paper by Atkin, Burke, and Greenwood investigates ways to improve the efficiency of an airport runway. The study considers the tradeoff between three mutually conflicting objectives-minimizing delay, complying with network departure constraints, and treating aircraft equitably-and provides insights for use in tuning the system to controller preferences.

The paper by Mirchandani, Li, and Hickman proposes a bus signal priority approach to cater for bus breakdown on a scheduled trip. In this paper, a macroscopic model that integrates bus signal priority with bus rescheduling is proposed. Computational results show that the joint consideration of bus signal priority and bus rescheduling effectively reduces the delay of the backup bus and decreases the delay costs.

We trust that these five papers provide timely reviews of recent methodological advances in public transport system scheduling and control. It is our hope that this special issue will inspire and stimulate new research initiatives and efforts in this still evolving field. 